\title{
ADDITIVITY OF MEASURE IMPLIES DOMINATING REALS
}

\author{
ARNOLD W. MILLER
}

\begin{abstract}
We show that additivity of measure $(A(m)$, the union of less than continuum many measure zero sets has measure zero) implies that every family $F \subseteq \omega^{\omega}$ of cardinality less than continuum is eventually dominated (this is the property $D)$. This yields as a corollary from known results that $A(m)+B(c) \rightarrow$ $A(c) . A(c)$ is the property that the union of less than continuum many first category sets has first category and $B(c)$ is the property that the real line is not the union of less than continuum many first category sets. Also, a new property of measure and category is introduced, the covering property, $C(\mathrm{~m})$ $(C(c))$, which says that for any family of measure zero (first category) sets of cardinality less than the continuum there is some measure zero (first category) set not covered by any member of the family. By dualizing the proof that $A(m) \rightarrow D$ we show that $w D \rightarrow C(m)$. The weak dominating property, $w D$, says that no small family contained in $\omega^{\omega}$ dominates every element of $\omega^{\omega}$.
\end{abstract}

Let $A(m)$ stand for the proposition that the union of less than continuum many measure zero sets has measure zero. Let $B(m)$ mean that the real line is not the union of less than continuum many measure zero sets. Let $U(m)$ stand for the proposition that every set of reals of cardinality less than the continuum has measure zero. And finally, let $C(m)$ stand for the proposition that there does not exist a family I of measure zero sets such that I has cardinality less than continuum and every measure zero set is covered by some element of $I$. (The letters $A, B, U, C$ are short for additivity, Baire, uniformity, covering.) $A(c), B(c), U(c)$ and $C(c)$ are defined similarly with "first category" (meager) replacing measure zero. Of course, these properties make sense for any ideal of sets of real numbers. The following implications hold for any nontrivial ideal ( $I$ should contain all singletons but not the whole real line):

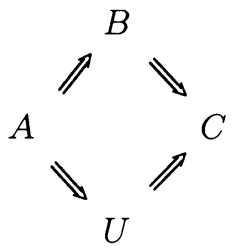

For example, to see that $U \Rightarrow C$ suppose $J \subseteq I$ covers every element of $I$. Then pick for each $J \in J$ some $x_{J} \notin J$. Then the set $\left\{x_{J}: J \in J\right\}$ will not be in $I$.

Next, let us introduce two properties concerning the eventually dominating order on $\omega^{\omega}$. The symbol " $\forall^{\infty}$ " stands for "for all but finitely many", and the symbol " $\exists^{\infty}$ " is short for "there exist infinitely many". The symbol $D$ (for dominating) stands for the property that for every $\mathcal{F} \subseteq \omega^{\omega}$ of cardinality less than continuum

Received by the editors September 27, 1982.

1980 Mathematics Subject Classification. Primary 03E35; Secondary 03E40. 
there exists $g \in \omega^{\omega}$ such that for all $f \in \mathcal{F}$,

$$
\forall^{\infty} n \in \omega \quad f(n)<g(n) .
$$

The property $w D$ (for weak dominating) is defined exactly the same, except the conclusion is

$$
\exists^{\infty} n \in \omega \quad f(n)<g(n) .
$$

Note that for every compact subset $K$ of $\omega^{\omega}$ there exists $f \in \omega^{\omega}$ such that $K \subseteq K_{f}=\left\{g \in \omega^{\omega}: \forall n g(n)<f(n)\right\}$ and also for every $f \in \omega^{\omega}, K_{f}$ is compact. Let $b$ stand for the $\sigma$-ideal generated by the compact subsets of $\omega^{\omega}$. The following implications are easy to verify:

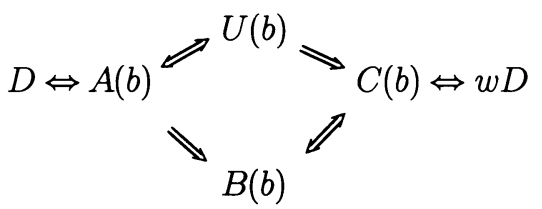

Tomek Bartoszyński (Warszawa) has shown that $D$ holds iff every union of less than continuum many compact subsets of $\omega^{\omega}$ is meager.

The following diagram adds the properties $D$ and $w D$ to the preceding diagram. All the nondotted implications hold for the ideal of measure zero sets and the ideal of first category sets. In addition, the dotted implications hold in the case of first category:

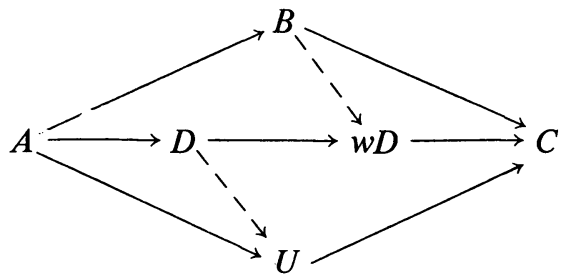

In the case of category, these implications are already known. To see that $A(c) \Rightarrow$ $D$ we have the following result.

Theorem 1 (Miller $[2,1.2]$ ). $A(c) \Leftrightarrow B(c)+D$.

The implication $w D \Rightarrow C(c)$ is a corollary to the proof of Theorem 1 . For completeness we will give the proof here.

Definitions. For $t$ a finite sequence in $2^{<\omega}$ let $[t]=\left\{x \in 2^{\omega}: t \subseteq x\right\}$.

LemMA 2. Suppose $M \subseteq N$ are models of $Z F C^{*}$ (some reasonable finite subtheory of $Z F C$ ) and for every nowhere dense closed set $A$ coded in $N$ there exists a first category set $B$ coded in $M$ such that $A \subseteq B$. Then for every $f \in N \cap \omega^{\omega}$ there exists a $g \in M \cap \omega^{\omega}$ such that for all $n<\omega^{\omega}, f(n)<g(n)$.

Proof. Suppose $f \in N \cap \omega^{\omega}$ and for every $g \in m \cap \omega^{\omega} \exists^{\infty} n g(n)<f(n)$. We may assume that $f$ is strictly increasing. Let $n_{k}=f(k)$. Note that for each $X \in[\omega]^{\omega} \cap$ $M, \exists^{\infty} k\left|\left[n_{k}, n_{k+1}\right] \cap X\right| \geq 2$. (Otherwise obtain $X \in[\omega]^{\omega} \cap M, \forall^{\infty} k, \mid\left[n_{k}, n_{k+1}\right] \cap$ $X \mid \leq 1$ and hence by throwing out finitely many elements of $X$ we can assume for 
all $k$. If $g$ enumerates $X$, then $g$ dominates $f$.) Now let $C=\left\{h \in 2^{\omega}: \forall k h\left(n_{k}\right)=1\right\}$. $C$ is obviously closed nowhere dense, and we claim that it is not covered by any first category set coded in $M$. To see this, let $\left\{C_{n}: n<\omega\right\}$ be an increasing sequence of closed nowhere dense subsets of $2^{\omega}$ coded in $M$. Construct (in $M$ ) an increasing sequence $m_{k}<\omega$ for $k<\omega$ as follows. Let $m_{0}=0$. Given $m_{k}$ choose $m_{k+1}$ so that for every $s \in 2^{m_{k}}$ there exists $t \in 2^{m_{k+1}}$ such that $t \subseteq s$ and $[t] \cap C_{k}=\varnothing$.

Since $\exists^{\infty} k \exists l n_{l} \leq m_{k}<m_{k+1} \leq n_{l+1}$, it is easy to see that for each $n, C \cap C_{n}$ is nowhere dense relative to $C$, hence $C$ is not covered by $\bigcup_{n<\omega} C_{n}$.

Theorem 3. $w D \Rightarrow C(c)$.

Proof. If $C(c)$ fails and $w D$ is true, then clearly by the reflection principle there are models $M \subseteq N$ of $\mathrm{ZFC}^{*}$ satisfying the hypothesis of Lemma 2, but not the conclusion.

REMARK. Of course, the amount of ZFC used in Lemma 2 is trivial and the reader can easily construct from a covering family of meager sets a dominating family in $\omega^{\omega}$. Nevertheless, we prefer this statement of Lemma 2 as it emphasizes the connection between our properties and extensions of models of set theory.

REMARK. The implication $A(c) \Rightarrow D$ is proved by using a sort of dual of Lemma 2. Namely, suppose $M \subseteq N$ are models of $\mathrm{ZFC}^{*}$ and there exists a first category set $C$ coded in $N$ which covers every nowhere dense closed set coded in $M$. Then there exist $f \in N \cap \omega^{\omega}$ such that or all $g \in M \cap \omega^{\omega}, \forall^{\infty} n g(n)<f(n)$.

Theorem 4 (Due to J. Cichoń And F. Galvin). Suppose $M \subseteq N$ are models of $Z F C^{*}$. Then the following are equivalent:

(1) Every nowhere dense closed set coded in $N$ is covered by a first category set coded in $M$.

(2) Every first category set coded in $N$ is covered by a first category set coded in $M$.

(3) Every nowhere dense closed set in $N$ is covered by a nowhere dense set coded in $M$.

Proof. Clearly $(3) \Rightarrow(1)$ and $(2) \Rightarrow(1)$. Define $x \approx y$ for $x, y \in 2^{\omega}$ iff $\forall^{\infty} n<$ $\omega x(n)=y(n)$ and define $X^{*}=\left\{y \in w^{\omega}: \exists x \in X x \approx y\right\}$ for $X \subseteq 2^{\omega}$. For any $X \subseteq 2^{\omega}, X$ has first category iff $X^{*}$ has first category. Also, for any $X \subseteq 2^{\omega}$ first category, there exists $P \subseteq 2^{\omega}$ nowhere dense such that $X \subseteq P^{*}$. To build such a $P$ proceed as follows. Consider forcing with the partial order $\mathbf{P}$ whose elements have the form $(n, P)$ where $n<\omega$ and $P \subseteq 2^{\omega}$ is a nowhere dense closed set. Order $\mathbf{P}$ by $(\bar{n}, \bar{P}) \leq(n, P)$ iff $\bar{n} \geq n, \bar{P} \supseteq P$, and for every $s \in 2^{n}$ if $[s] \cap \bar{P} \neq \varnothing$, then $[s] \cap P \neq \varnothing$ (i.e. $(n, P)$ says the perfect set we are building looks like $P$ up to level $n$ ). If $G$ is $\mathbf{P}$-generic over $M$, then let $P_{G}$ be the closure of $\bigcup\{P: \exists n(n, P) \in G\} . P_{G}$ is a nowhere dense closed set such that for all $P$ nowhere dense closed sets coded in $M$ there exists $n$ such that for all $x \in P$ there exists $y \in P_{G}, x \uparrow(\omega-n)=y \uparrow(\omega-n)$. Thus if $P=P_{G}$ for some $G \mathbf{P}$-generic over a countable $M$ containing a code for $X$, then $X \subseteq P^{*}$. To see that $(1) \Rightarrow(2)$ suppose that $X$ is first category and coded in $N$. Let $P$ be nowhere dense and $X \subset P^{*}$. By (1) there exists $Y$ first category coded in $M$ and $P \subseteq Y$. Then $Y^{*}$ is first cateogry, coded in $M$, and $X \subseteq Y^{*}$.

To see that $(1) \Rightarrow(3)$ first note that by Lemma 2 every $f \in N \cap \omega^{\omega}$ is dominated by some $g \in M \cap \omega^{\omega}$. Suppose $P$ is closed nowhere dense and coded in $N$. Since $P$ is covered by a first category set coded in $M$ we can find (in $M$ ) a set $\left\{r_{n}: n<\right.$ 
$\omega\} \subseteq 2^{\omega}$ which is dense and disjoint from $P$. In $N$ there exists $f \in \omega^{\omega}$ such that for all $n<\omega$

$$
\left[r_{n} \uparrow f(n)\right] \cap P=\varnothing .
$$

Let $g \in M \cap \omega^{\omega}$ dominate $f$ and note that

$$
G=\bigcup_{n<\omega}\left[r_{n}\lceil g(n)]\right.
$$

is an open dense set coded in $M$ and disjoint from $P$.

We now start the proof of the main result of this paper, $A(m) \rightarrow D$ and $w D \rightarrow$ $C(m)$. For $g \in \omega^{\omega}$ let

$$
H_{g}=\left\{x \in 2^{\omega} \mid \exists^{\infty} n x \uparrow[g(n), g(n)+n) \equiv 0\right\} .
$$

Note that $H_{g}$ is a Borel set of zero-measure with a code in any model of ZFC* containing $g$. For $G \subseteq 2^{\omega}$ an open set and $n<\omega$, define $G_{n}=\bigcup\left\{[s]: s \in 2^{n},[s] \subseteq\right.$ $G\}$. Define a decreasing sequence $\epsilon_{k}>0$ so that

$$
\sum_{k=0}^{\infty} 2^{k^{2}} \epsilon_{k}<\frac{1}{2} \text {. }
$$

For $G \subseteq 2^{\omega}$ open, define $f_{G} \in \omega^{\omega}$ strictly increasing so that for all $n<\omega$ the measure of $G-G_{f_{G}(n)}$ is less than $\epsilon_{n}$.

Lemma 5. Suppose $G$ is an open set of measure less than $\frac{1}{2}$. If $H_{g} \subseteq G$, then $\forall \forall^{\infty} n g(n)<f_{G}(n)$.

Proof. Suppose $\exists^{\infty} n f_{G}(n) \leq g(n)$ and let $h \in \omega^{\omega}$ be strictly increasing and for each $n, f_{G}(h(n)) \leq g(h(n))$ and $g(h(n))+h(n) \leq g(h(n+1))$. (Thus the intervals $[g(h(n)), g(h(n))+h(n))$ are disjoint.)

For $x \in 2^{\omega}$ and $n<m<\omega, x \uparrow[n, m) \equiv 0$ means that $x(l)=0$ for all integers $l$ with $n \leq l<m$. Let

$$
P=\left\{x \in 2^{\omega} \mid \forall n x \uparrow[g(h(n)), g(h(n))+h(n)) \equiv 0\right\} .
$$

Now $P$ is a closed subset of $H_{g}$ and we will show that $P$ is not contained in $G$, a contradiction. Define the clopen set $C_{k}$ for each $k<\omega$ by $C_{-1}=2^{\omega}$ and

$$
C_{k}=C_{k-1} \cap\left\{x \in 2^{\omega}: x \uparrow[g(h(k)), g(h(k))+h(k)) \equiv 0\right\} .
$$

Now $P=\bigcap_{k<\omega} C_{k}$ and thus by compactness it is enough to show that for all $k<\omega$, $\neg\left(C_{k} \subseteq G\right)$. This will be shown by proving that $\mu\left(G \cap C_{k}\right)<\mu\left(C_{k}\right)$.

Claim. $\mu\left(G \cap C_{k}\right) \leq\left(1 / 2^{h(k)}\right) \mu\left(G \cap C_{k-1}\right)+\epsilon_{h(k)}$.

Proof.

$$
\begin{gathered}
\mu\left(G \cap C_{k}\right) \leq \mu\left(G-G_{g(h(k))}\right)+\mu\left(G_{g(h(k))} \cap C_{k}\right), \\
\mu\left(G-G_{g(h(k))}\right) \leq \epsilon_{h(k)}, \\
\mu\left(G_{g(h(k))} \cap C_{k}\right)=\frac{1}{2^{h(k)}} \mu\left(G_{g(h(k))} \cap C_{k-1}\right), \\
\frac{1}{2^{h(k)}} \mu\left(G_{g(h(k))} \cap C_{k-1} \leq \frac{1}{2^{h(k)}} \mu\left(G \cap C_{k-1}\right) .\right.
\end{gathered}
$$


Formulae (1) and (4) are trivial. Formula (2) follows from the fact that $f_{G}(h(k)) \leq$ $g(h(k))$. So $G-G_{g(h(k))} \subseteq G-G_{f_{G}(h(k))}$ which has measure less than $\epsilon_{h(k)}$. Formula (3) is true because

(a) $G_{g(h(k))}=\bigcup\left\{[s]: s \in 2^{g(h(k))},[s] \subseteq G\right\}$ and

(b) $C_{k}=C_{k-1} \cap\left\{x \in 2^{\omega}: x \uparrow[g(h(k)), g(h(k))+h(k)) \equiv 0\right\}$.

Thus $G_{g(h(k))} \cap C_{k-1}$ is a union of $[s]$ for some $s \in 2^{g(h(k))}$. From the four formulae the claim is easily proved.

Now for each $k<\omega$, we have $\mu\left(C_{k}\right)=\mu\left(C_{k-1]}\right) / 2^{h(k)}$. Thus from the claim

$$
\frac{\mu\left(G \cap C_{k}\right)}{\mu\left(C_{k}\right)} \leq \frac{\mu\left(G \cap C_{k-1}\right)}{\mu\left(C_{k-1}\right)}+\frac{\epsilon_{h(k)}}{\mu\left(C_{k}\right)} .
$$

Now

By induction

$$
\frac{1}{\mu\left(C_{n}\right)}=2^{\sum_{i=0}^{n} h(i)} \leq 2^{(h(n))^{2}} .
$$

$$
\frac{\mu\left(G \cap C_{k}\right)}{\mu\left(C_{k}\right)} \leq \mu(G)+\sum_{i=0}^{k} \epsilon_{h(i)} 2^{h(i)^{2}} .
$$

But by the choice of the $\epsilon_{n}$, this is less than 1 so $G$ does not cover $C_{k}$.

LemMA 6. Suppose $M \subseteq N$, are models of ZFC* and for every measure zero set $A$ coded in $N$ there exists an open set $B$ of measure less than $\frac{1}{2}$ coded in $M$ such that $A \subseteq B$. Then for every $g \in N \cap \omega^{\omega}$ there exists $f \in M \cap \omega^{\omega}$ such that for all $n<\omega$, $g(n)<f(n)$.

Proof. Suppose $g \in N \cap \omega^{\omega}$ and let $H_{g}$ be the measure zero set constructed above. If $G$ is an open set of measure less than $\frac{1}{2}$ coded in $M$ such that $H_{g} \subseteq G$, then we have by Lemma 5 that $f_{G} \in M \cap \omega^{\omega}$ eventually dominates $g$.

LeMmA 7. Suppose $M \subseteq N$ are models of $Z F C^{*}$ and for every $f \in N \cap \omega^{\omega}$, there exists $g \in M \cap \omega^{\omega}$ such that $\exists^{\infty} n f(n)<g(n)$. Then there does not exist an open $G$ coded in $N$ of measure less than $\frac{1}{2}$ such that $G$ covers every measure zero set coded in $M$.

Proof. Suppose $G$ is an open set coded in $N$ of measure less than $\frac{1}{2}$. Then let $g \in M \cap \omega^{\omega}$ such that $\exists^{\infty} n f_{G}(n)<g(n)$. By Lemma 5, G does not cover $H_{g}$.

Theorem 8. $A(m) \rightarrow D$ and $w D \rightarrow C(m)$.

Proof. This follows from Lemmas 6 and 7 and the reflection principle.

REMARK. In light of this theorem it is tempting to conjecture that $A(m) \leftrightarrow$ $B(m)+D$. But it was pointed out to me by Cichon and Kamburelis that this is not true. If one starts with a model in which the continuum is $\omega_{2}$ and $D$ holds and then adds $\omega_{2}$ random reals, then in the resulting model it is not difficult to show that $B(m)+D+\neg U(m)$ is true.

Theorem 9. $A(m)+B(c) \rightarrow A(c)$.

Proof. This is a corollary of Theorems 8 and 1 .

REMARK. Up until now the only known implications among the properties $A, B$ and $U$ were that $A \rightarrow B$ and $A \rightarrow U$ and Rothberger's Theorem [4] that $B(c) \rightarrow$ $U(m)$ and $B(m) \rightarrow U(c)$. 
Remark. Recently Bartosyński showed that, in fact, $A(m) \rightarrow A(c)$. He used in part the results of this paper. Working independently Raissonier and Stern obtained the result of this paper and also of Bartosyński. Bartosyński also obtained the characterization of $A(m)$ :

$$
\begin{aligned}
A(m) \leftrightarrow & \forall \mathcal{F} \in\left[\omega^{\omega}\right]^{<c} \exists h_{n} \in[\omega]^{n} \text { for } n<\omega \\
& \forall f \in \mathcal{F} \forall_{n}^{\infty} f(n) \in h_{n} .
\end{aligned}
$$

Kamburelis and Krawczyk showed that in the domainating real plus random real iteration, $\neg A(m)+A(c)+B(m)$ holds. They used the above characterization to see $\neg A(m)$. Several people have also noticed that $C(c) \rightarrow C(m)$ by dualizing the proof that $A(m) \rightarrow A(c)$.

REMARK. Both $\neg C(c)+\neg C(m)$ and $\neg U(c)+\neg U(m)+w D$ are consistent. The two models concerned are discussed in Miller [2, second to last paragraph on $\mathrm{p}$. 107]. The iterated Sacks real model satisfies $\neg C(c)+\neg C(m)$. If one starts with a model in which $D$ holds and $\mathrm{CH}$ fails and then iteratively adds $\omega_{1}$ random reals with finite supports, then in the resulting model both $U(m)$ and $U(c)$ fail; however $w D$ is true ( $w D$ follows from the results in $\S 4$ of Miller [2]).

REMARK. Here is an updated version of the chart from Miller [3]. That chart was first made by Kunen [1].

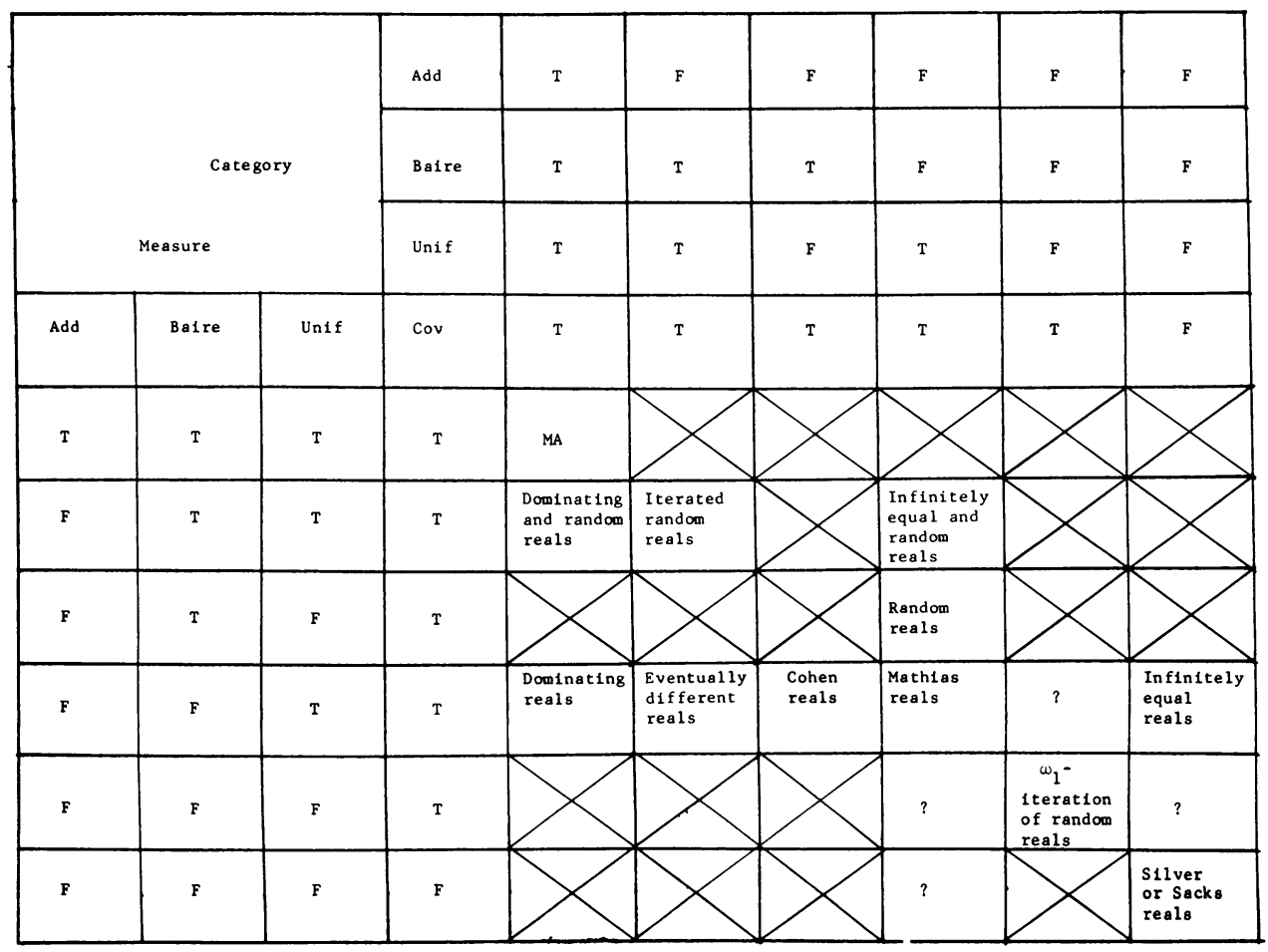

REMARK. I want to thank Cichon and Kamburelis for some very helpful suggestions on the contents of this paper. 


\section{REFERENCES}

1. K. Kunen, Random and Cohen reals, Handbook of Set Theoretic Topology, North-Holland, Amsterdam, 1984.

2. A. Miller, Some properties of measure and category, Trans. Amer. Math. Soc. 266 (1981), 93-114; Corrections and Additions, ibid. 271 (1982), 347-348.

3. $\ldots$ A characterization of the least cardinal for which the Baire category theorem fails, Proc. Amer. Math. Soc. 86 (1982), 498-502.

4. F. Rothberger, Eine Äquivalenz zwischen der Kontinuumhypothese und der Existenz der Lusinschen und Sierpińskischen Mengen, Fund. Math. 30 (1938), 215-217.

Department of Mathematics, The University of Texas, Austin, Texas 78712 